\title{
Low-cost fully integrated fiber Bragg grating interrogation system
}

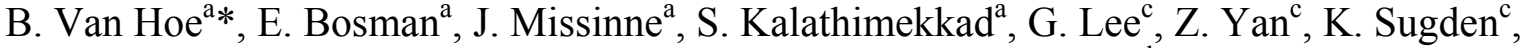 \\ D.J. Webb ${ }^{c}$, G. Van Steenberge ${ }^{\mathrm{a}}$, P. Van Daele ${ }^{\mathrm{b}}$ \\ ${ }^{a}$ Centre for Microsystems Technology (CMST), Elis Department, Ghent University-imec, Belgium \\ ${ }^{\mathrm{b}}$ Centre for Microsystems Technology (CMST), Intec Department, Ghent University-imec, Belgium \\ ${ }^{c}$ Photonics Research Group, Aston University, United Kingdom
}

\begin{abstract}
Fiber Bragg gratings can be used for monitoring different parameters in a wide variety of materials and constructions. The interrogation of fiber Bragg gratings traditionally consists of an expensive and spacious peak tracking or spectrum analyzing unit which needs to be deployed outside the monitored structure. We present a dynamic low-cost interrogation system for fiber Bragg gratings which can be integrated with the fiber itself, limiting the fragile optical in- and outcoupling interfaces and providing a compact, unobtrusive driving and read-out unit. The reported system is based on an embedded Vertical Cavity Surface Emitting Laser (VCSEL) which is tuned dynamically at $1 \mathrm{kHz}$ and an embedded photodiode. Fiber coupling is provided through a dedicated $45^{\circ}$ micromirror yielding a $90^{\circ}$ in-the-plane coupling and limiting the total thickness of the fiber coupled optoelectronic package to $550 \mu \mathrm{m}$. The red-shift of the VCSEL wavelength is providing a full reconstruction of the spectrum with a range of $2.5 \mathrm{~nm}$. A few-mode fiber with fiber Bragg gratings at $850 \mathrm{~nm}$ is used to prove the feasibility of this low-cost and ultra-compact interrogation approach.
\end{abstract}

Keywords: fiber Bragg grating, dynamic, low-cost, integrated, interrogation, VCSEL, flexible optoelectronic package

\section{INTRODUCTION}

Although the use of fiber Bragg gratings as sensing elements for strain, temperature and pressure is widespread and silica fibers with Bragg gratings are commercially available through multiple companies [1] [2], the interrogation units are mostly expensive and spacious. Moreover, traditional fiber Bragg grating interrogation systems often come with a difficult trade-off between the possibility of performing dynamic measurements limited to peak tracking, and only static measurements enabling full spectrum reconstruction. A lot of important information can be lost this way, especially when doing impact or vibration tests. Transverse strain for example can cause the FBG spectra to shift but also to spread.

Interesting interrogation alternatives have been suggested by different research institutes and universities. Dynamic measurements capturing the FBG spectral data at rates of $100 \mathrm{kHz}$ are reported using a broadband source and a tunable photodetector [3]. Peak-tracking systems have been developed with highly sensitive detection of periodic or transient events up to the MHz range [4]. All these systems however still require large and expensive driving and read-out units. We present a compact and low-cost alternative based on a VCSEL-photodetector interrogation scheme which can be embedded in an optical sensing skin providing a fully embedded fiber sensing interrogation approach. An ultra-thin package is developed to integrate the optoelectronics in thin flexible foils and a dedicated in-the-plane coupling technology is reported to come up with photonic skin-compliant driving optoelectronic units. The final sensing sheet consequently consists of a fiber with Bragg gratings and fiber coupled driving and read-out optoelectronics, schematically shown in Figure 1. A fully embedded polymer skin is the result providing a flexible unobtrusive sheet that can be wrapped around, embedded in, attached and/or anchored to irregularly shaped and/or moving objects. Modulating the VCSEL provides a dynamic reconstruction of the fiber Bragg grating spectrum avoiding any loss of information in the event of peak deformation.

*For more information, contact: Bram.VanHoe@elis.ugent.be; phone + 32 (0)9 26453 70; fax + 32 (0) 92645374 ; http://www.cmst.be

Third Asia Pacific Optical Sensors Conference, edited by John Canning, Gangding Peng,

Proc. of SPIE Vol. 8351, 83510U · C 2012 SPIE · CCC code: 0277-786X/12/\$18 · doi: 10.1117/12.914253 


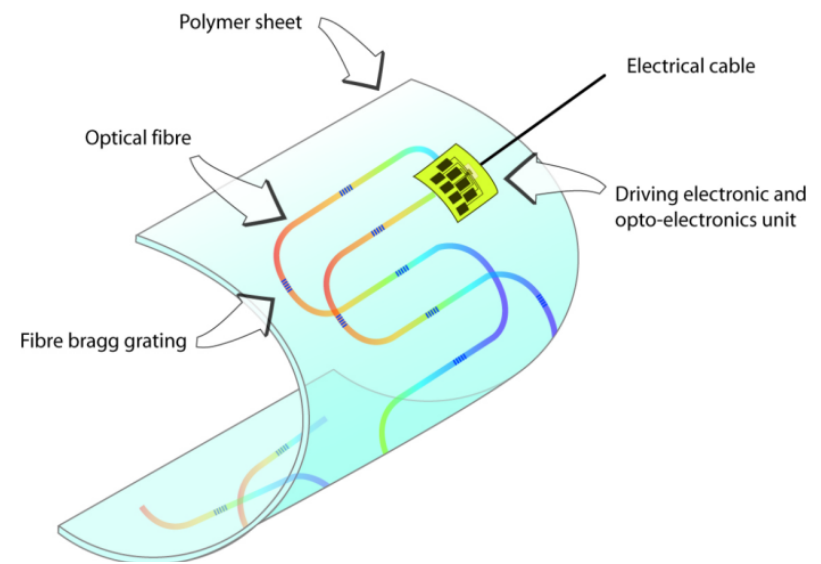

Figure 1: Sensing sheet with fiber sensors, driving and read-out optoelectronics and fiber coupling technology integrated in a flexible polymer sheet.

\section{INTERROGATION SCHEME}

The interrogation scheme we present is schematically shown in

Figure 2. A VCSEL is used as a fiber optic driving unit and a photodiode is used to read-out the transmitted power. An electrical driving unit is needed to modulate the electrical current in the VCSEL and this way enabling the VCSEL wavelength red-shifting. Depending on the separation of the different Bragg gratings, this system has (limited) possibility to read-out multiplexed fiber sensing points. A data acquisition board linked to a computer is used to store and visualize the photocurrent through the integrated photodiode.

Figure 3 shows a basic example of the interrogation scheme. In Figure 3 (a), the VCSEL is modulated with a sawtooth signal and the optical power and wavelength are varying accordingly. Figure 3 (b) shows the response of the photodetector current when a Bragg grating is measured in transmission. The photocurrent is depicting the sawtooth driving signal and the grating filter characteristic. Combining the data in Figure 3 (b) with the data from a VCSEL calibration measurement enables us to filter the sawtooth signal and isolate the grating response in the photocurrent signal (Figure 3 (c)). Assuming the VCSEL is modulated using a frequency $\mathrm{f}$, each $1 / \mathrm{f}$ seconds a new full spectrum reconstruction is available. Assigning a color to the relative wavelength intensity results in a complete spectral reconstruction (Figure 3 (d)) in which the intensity is depicted for each wavelength every $1 / \mathrm{f}$ seconds.

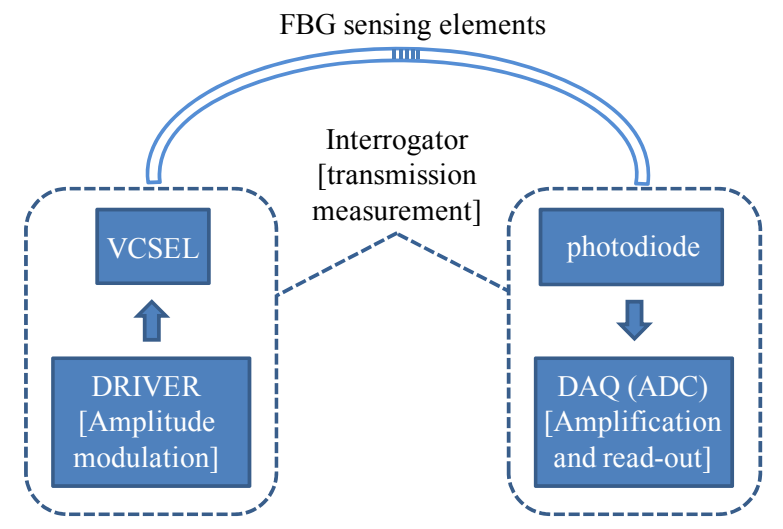

Figure 2: Interrogation system block diagram. 
(a)

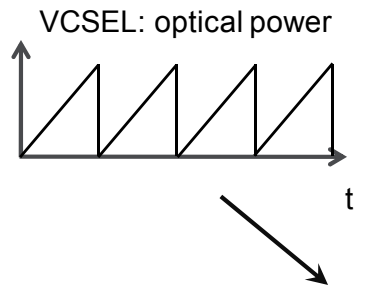

VCSEL: $\lambda$ fundamental mode

(b)
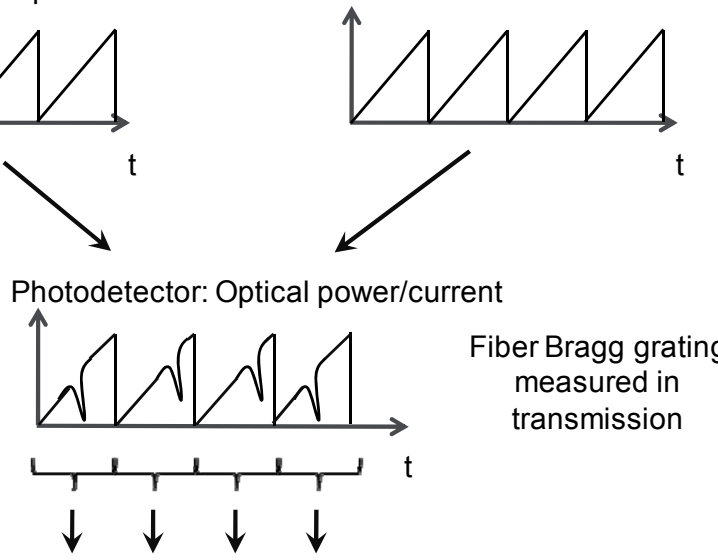

Fiber Bragg grating

measured in

transmission

(c)

$\lambda$

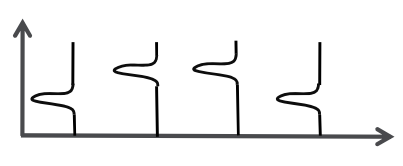

$\lambda$

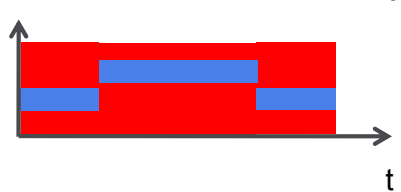

Color $=\lambda$ intensity

Figure 3: Interrogation scheme example. (a) The VCSEL is modulated with a sawtooth signal. (b) A Bragg grating is measured in transmission. The photocurrent is depicting the sawtooth driving signal and the grating filter characteristic. (c) Combining calibration measurements and the functional signal leads to (d) a full spectral reconstruction.

\section{FLEXIBLE OPTOELECTRONIC DRIVING AND READ-OUT PACKAGE}

The driving and read-out optoelectronics consist of two ultra-thin flexible optoelectronic packages with compact planar fiber coupling plugs. Driving the fiber sensor is done using a single-mode GaAs VCSEL chip and the read-out is performed using a GaAs photodetector array chip. Processing the flexible package starts on a rigid glass carrier and ends with a release from this carrier. The embedding material for the optoelectronic package needs to be transparent and provides a basis for a mechanically reliable and flexible entity. Therefore the transparent epoxy material SU-8 is chosen. This SU-8 material is sandwiched in between two strong and highly flexible polyimide layers. By minimizing the thickness of the stack, even the SU-8 layers become very flexible. The chip is positioned in the middle of the stack, where tensile and pressure forces are the lowest during bending of the package. The original chip thickness of the VCSELs and photodiodes is $125 \mu \mathrm{m}$ and they are consecutively lapped and polished down to $25 \mu \mathrm{m}$ maintaining their electrical and optical characteristics. A new electrical back contact is applied by AuGeNiAu sputtering/evaporating.

A detailed overview of the different process steps for the fabrication of the flexible optoelectronic package is shown in Figure 4. The complete package is fabricated on top of a rigid glass carrier and released after processing (f). On the glass carrier, a polyimide layer and an SU-8 layer are spincoated consecutively (a). In between those two layers, a local heat sink consisting of a copper island is deposited. The SU-8 material on top of the heat sink is removed using laser ablation (b), creating a cavity in which the optoelectronic devices (VCSEL and photodiode) are mounted using an electrically conductive glue (c,d). A second spin-coated SU-8 layer covers the chip and laser ablated vias open the contact pads to the embedded chip (e). A galvanic fan-out is deposited and structured and finally another thin SU-8 layer and the top polyimide layer are spincoated (f). Releasing the flexible package from the processing carrier is done by laser cutting. More details on this embedding process can be found in [5].

The thickness of the total chip package is limited to only $50 \mu \mathrm{m}$. The optical characteristics of an embedded single-mode $850 \mathrm{~nm}$ VCSEL are shown in Figure 5 proving the fully functional embedded optoelectronic device. The total tuning range of the central VCSEL wavelength, a key parameter for fiber Bragg interrogation, is $2.5 \mathrm{~nm}$. 


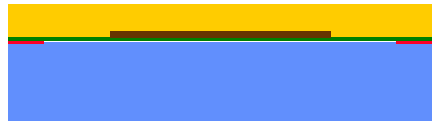

(a) Polymer layer deposition on rigid glass carrier

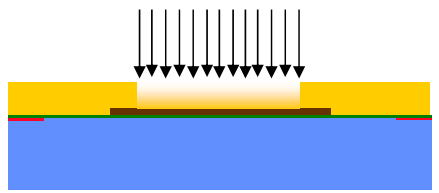

(b) Laser patterning cavity

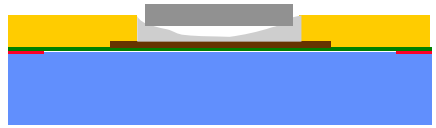

(c) Face-up chip placement and fixation

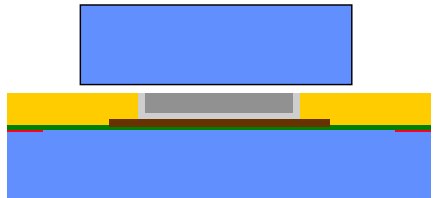

(d) Chip leveling inside cavity

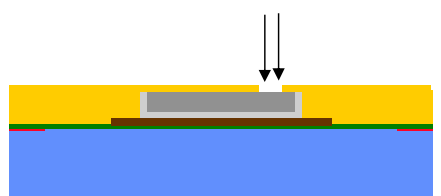

(e) Laser blind via drilling to chip contacts

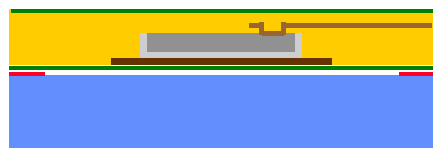

(f) Electrical contacting, polymer layer deposition and release from glass carrier

Figure 4: Process flow flexible embedded optoelectronic components.
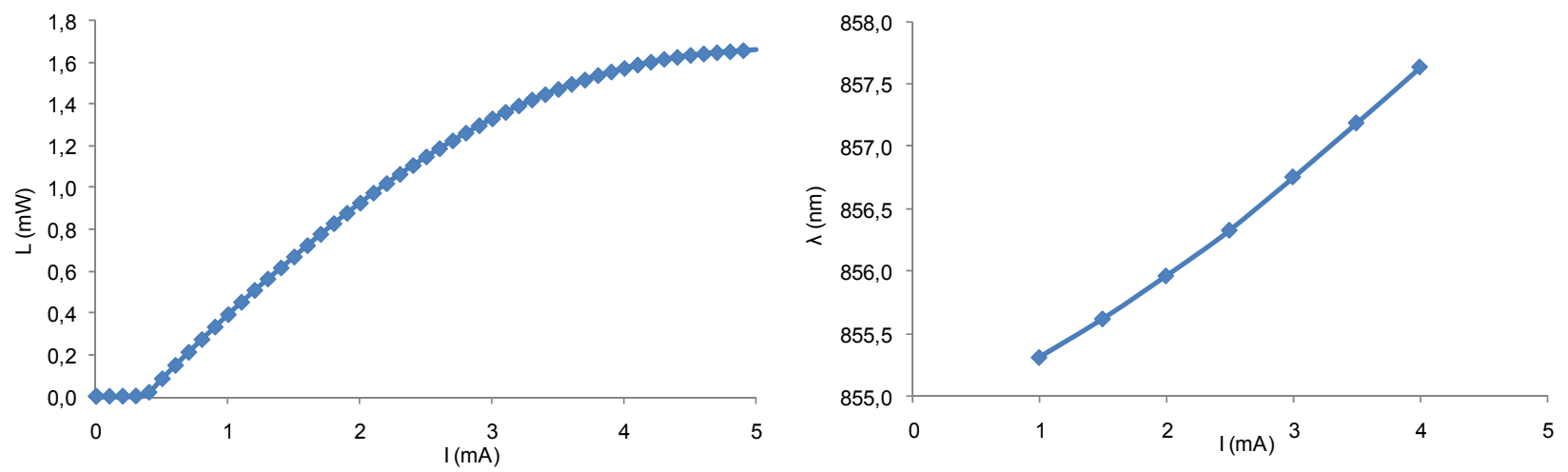

Figure 5: Optical (power and wavelength) characteristics of thinned, embedded single-mode $850 \mathrm{~nm}$ VCSELs.

\section{FIBER COUPLING}

To come up with a skin-compliant fiber coupled driving unit, a dedicated in-the-plane coupling scheme is developed. This coupling scheme consists of a PMMA plug $(500 \mu \mathrm{m}$ thickness) with a U- or V-groove to clamp the fiber, schematically shown in Figure 6 . By using a dedicated $45^{\circ}$ clamping master tool and different lapping/polishing techniques, a $45^{\circ}$ mirror facet is applied directly on the coupling fiber in the PMMA plug limiting the optical path and consequently minimizing the losses (Figure 6). The fiber is fixed with a special index-matched UV-glue and the reflecting layer is applied by chemically evaporating a thin (few $100 \mathrm{~nm}$ ) layer of gold. When the coupling plug is mounted on top of the VCSEL package, the vertically emitted light is reflected on the gold surface through the coupling plug in the optical fiber. A detailed description of this fabrication process can be found in [6].
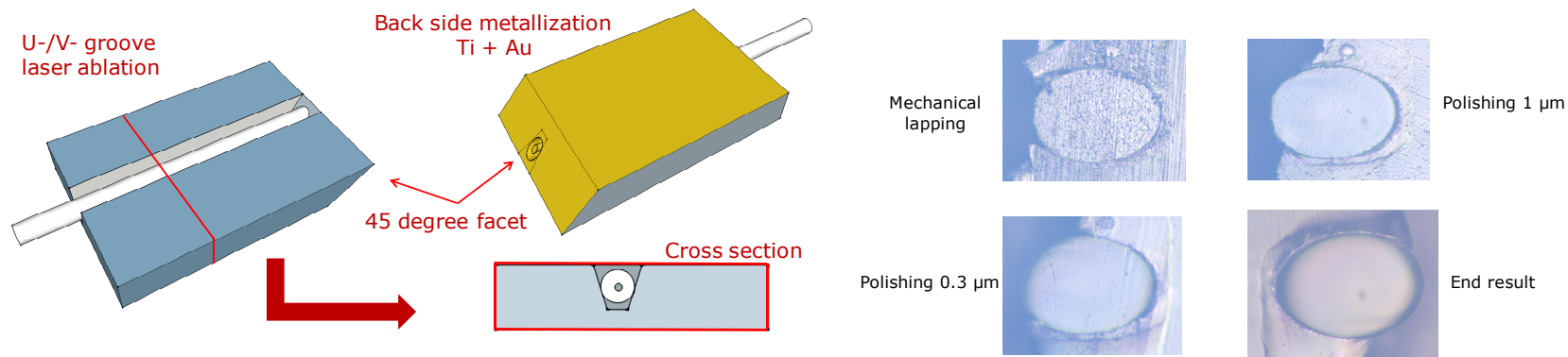

Figure 6: Schematic view of coupling scheme (left). Microscope view of $45^{\circ}$ polished fiber facet (right).

The end result is a smooth fiber facet of optical quality with an average surface roughness of $23 \mathrm{~nm}$ on a $50 \mu \mathrm{m} \times 50 \mu \mathrm{m}$ area. Coupling losses inherent to the fiber coupling plugs are measured using a commercially available fiber coupled single-mode laser diode at $850 \mathrm{~nm}$ and a resulting coupling loss of $1 \mathrm{~dB}$ is found. Figure 7 is depicting a resulting 
flexible fiber coupled optoelectronic package with a total thickness of $550 \mu \mathrm{m}$, enabling the embedding in photonic skins (typical thickness is $1 \mathrm{~mm}$ ) and the combination of driving and read-out devices in a fully embedded photonic skin. Experimental coupling losses of the fiber coupled single-mode VCSELs are limited to 2 to $3 \mathrm{~dB}$. Coupling integrated photodetectors results in experimental coupling losses around $1 \mathrm{~dB}$.
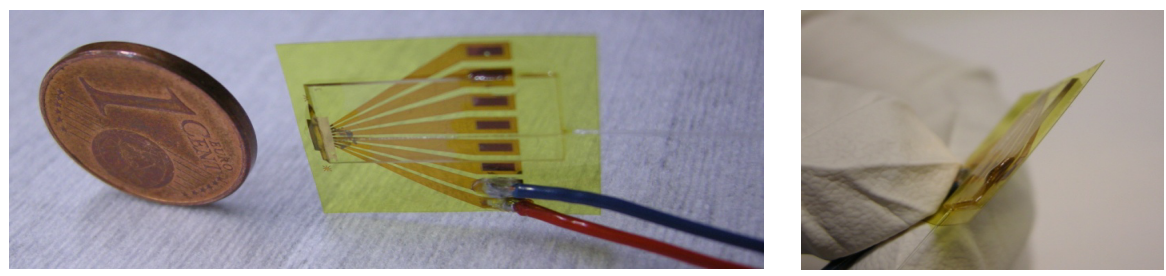

Figure 7: Flexible fiber coupled optoelectronic package with a total thickness of $550 \mu \mathrm{m}$.

\section{FULLY EMBEDDED SYSTEM}

To characterize the fully embedded system, a dedicated set-up is built in which a few-mode silica fiber with a Bragg grating is used. As a reference, the transmission spectrum of this grating is recorded using a compact fiber pigtailed Exalos Superluminescent light-emitting diode (SLD) and an Agilent 86142B optical spectrumanalyzer (OSA), shown in Figure 8 . The sensing fiber is then clamped between two stages accommodated with mircrosrews to apply a uniform strain on the Bragg grating.

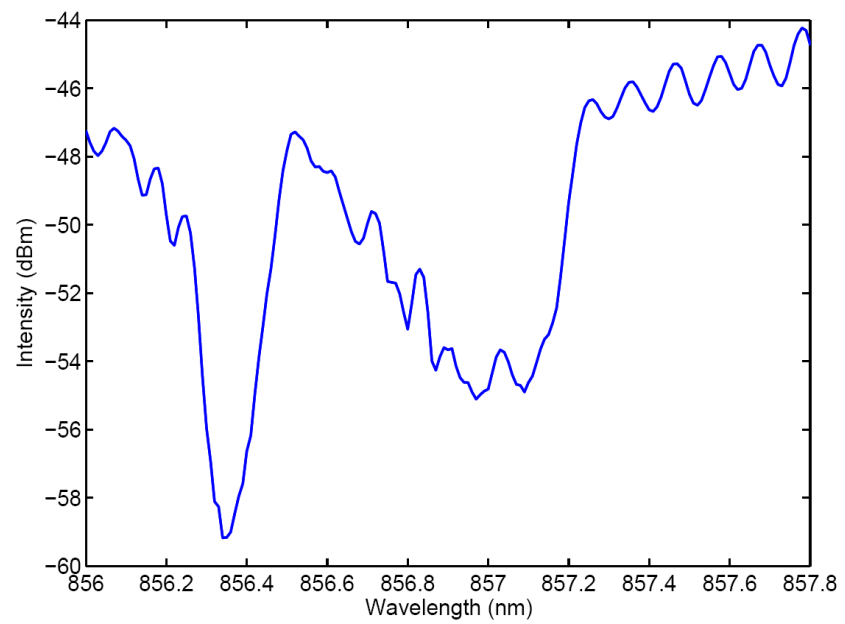

Figure 8: Fiber Bragg grating in transmission using Exalos SLD and an Agilent 86142B OSA.

The fully embedded interrogation scheme is discussed in detail in section 2 and consists of an embedded VCSEL which is actively coupled to a single-mode fiber to maximize the power coupled to the fiber Bragg grating and an embedded detector which is fiber coupled as described in section 4 (Figure 9). Driving the VCSEL is done using a $1 \mathrm{kHz}$ sawtooth current signal and the VCSEL is modulated between $2 \mathrm{~mA}$ and $4 \mathrm{~mA}$. A linear relation between electrical current and VCSEL wavelength is assumed. The photocurrent signal (a measure of the transmitted optical power) is amplified in a negative feedback loop and filtered to clear the signal from high frequency noise. A National Instruments data acquisition (DAQ) board is used to read-out and store the functional photocurrent data and the DAQ read-out frequency is set at $10^{5}$ samples per second. Consequently, every millisecond a new spectral waveform is available and each waveform consists of 100 data points. 

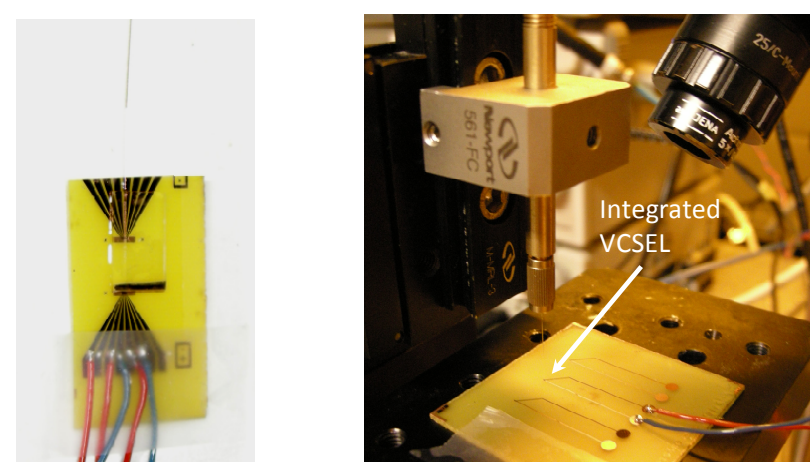

Figure 9: (left) Embedded and fiber coupled photodetector and (right) a single-mode silica fiber aligned on top of an integrated thinned VCSEL chip.

The total VCSEL tuning range is $1.783 \mathrm{~nm}$ yielding a spectral resolution accuracy of $17.83 \mathrm{pm}$. Table 1 is summarizing the test set-up driving parameters. Increasing the VCSEL modulation or photodetector sampling frequency can further enhance time and spectral resolution of the system. The upper limit for the modulation frequency in this simple approach was experimentally determined around $20 \mathrm{kHz}$. Beyond this limit, the wavelength-current relationship of the VCSEL starts to fade. By using pulse width modulation or other more advanced signal processing techniques, one could further enhance the resolution values.

Table 1: Parameter overview of the embedded interrogation test set-up.

\begin{tabular}{|c|c|}
\hline Parameter & Value \\
\hline Addressed dynamic range VCSEL & $\begin{array}{c}\lambda_{\text {fundamental, start }}=855.877 \mathrm{~nm} \\
\lambda_{\text {fundamental, end }}=857.660 \mathrm{~nm}\end{array}$ \\
\hline Driving frequency VCSEL & $1 \mathrm{kHz}$ \\
\hline Read-out frequency photodetector & $100 \mathrm{kHz}$ \\
\hline Read-out filter cut-off frequency & $1 \mathrm{MHz}$ \\
\hline
\end{tabular}

A calibration measurement is necessary in order to filter the optical power fluctuations and to come up with relative wavelength intensities. During the functional test, a manual strain of 0.954 mstrain is applied by loading the fiber with a microscrew with a resolution of $10 \mu \mathrm{m}$. The relative wavelength intensities are color plotted as a function of time (measurement principles are discussed in section 2) in Figure 10. This figure displays a dynamic measurement of the fiber Bragg grating during manual straining and releasing of the fiber. The different straining and releasing steps are clearly visible. A more detailed view of $t=14 \mathrm{~s}$ to $t=15 \mathrm{~s}$ (containing 1000 spectral measurements) is shown in Figure 11.

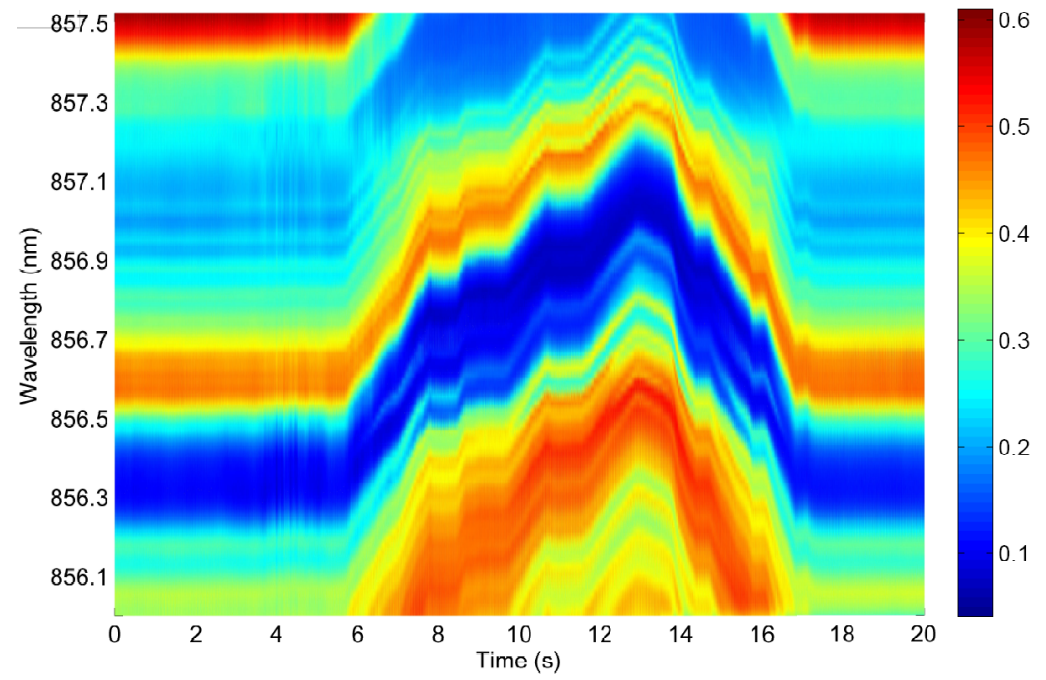

Figure 10: Dynamic measurement of the fiber Bragg grating using the fully embedded system [wavelength intensity vs. time]. 


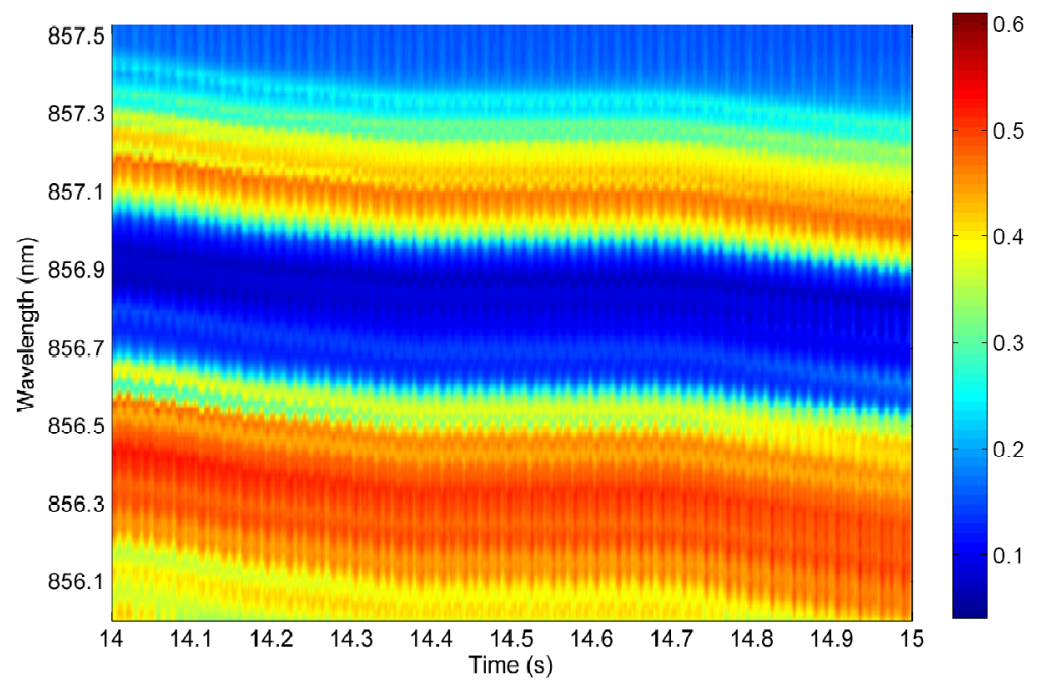

Figure 11: Dynamic measurement of the fiber Bragg grating using the fully embedded system [wavelength intensity vs. time], detailed view.

In order to experimentally verify the strain which is applied, the minimum wavelength intensity is tracked. A maximum wavelength shift of $0.760 \mathrm{~nm}$ is obtained at $\mathrm{t}=12.96 \mathrm{~s}$. Silica fibers have a typical strain sensitivity of $1 \mathrm{pm} / \mu$ strain [7] translating the experimental wavelength shift to 0.760 mstrain. Comparing this value to the theoretical strain of 0.954 mstrain indicates the occurrence of partial slippage of the fiber in the fiber clamps as the displacement is not fully transferred into strain on the fiber.

More advanced analysis of the spectral response of the fiber Bragg grating is now possible on a very accurate scale, both in time and wavelength domain. Different snapshots of the optical spectrum are shown in Figure 12 and Figure 13 . One can extract the shift in peak wavelength but also other information, not measurable with traditional peak-tracking systems, such as peak deformation or the introduction of birefringence (Figure 10, $\mathrm{t}=7 \mathrm{~s}$ and snapshot in Figure 13) is visible.

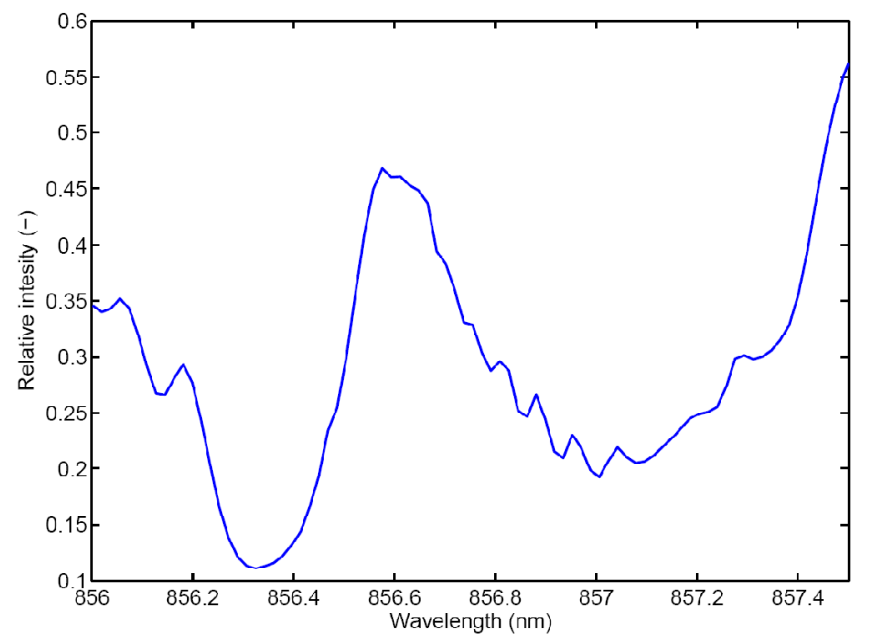

Figure 12: Snapshot of the spectral information at $\mathrm{t}=1 \mathrm{~s}$ [intensity vs. wavelength]. 


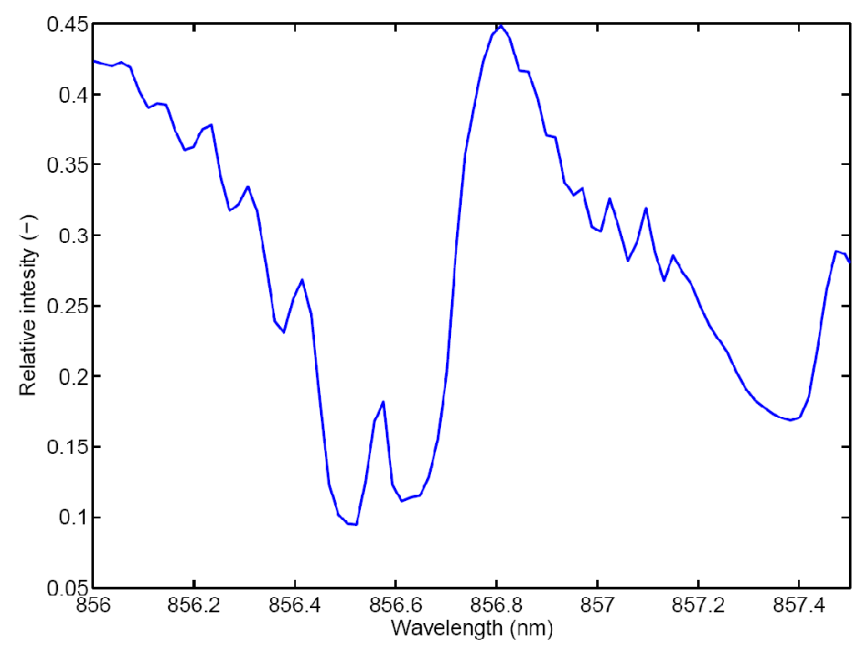

Figure 13: Snapshot of the spectral information at $\mathrm{t}=7 \mathrm{~s}$ [intensity vs. wavelength].

\section{CONCLUSION}

We have demonstrated a low-cost and compact fiber sensing interrogation approach using a VCSEL and photodiode, packaged and fiber coupled in an ultra-thin flexible polymer stack. A few-mode silica sensing fiber in combination with a $1 \mathrm{kHz}$ modulation of the VCSEL was used for the characterization tests, providing a continuous reconstruction of the fiber Bragg grating spectral response.

\section{ACKNOWLEDGEMENTS}

This work is partially conducted in the framework of the project PHOSFOS [8], Photonic Skins for Optical Sensing (funded within the EU-FP7 program). The research of B. Van Hoe is supported by a Ph.D grant of the Institute for the Promotion of Innovation through Science and Technology in Flanders (IWT-Vlaanderen).

\section{REFERENCES}

[1] FOS\&S Fiber Optic Sensing, http://www.fos-s.be

[2] Technobis Fibre Technologies - Fibre Optic Sensing, http://www.tft-fos.com

[3] T. Vella, S. Chadderdon, R. Selfridge, S. Schultz, S. Webb, C. Park, K. Peters and M. Zikry, "Full-spectrum interrogation of fiber Bragg gratings at $100 \mathrm{kHz}$ for detection of impact loading", Meas. Sci. Technol. 21, 094009 (2010).

[4] L. U. Kempen, E. A. Mendoza and I. F. Saxena, "Self-compensated high-speed FBG interrogation using closedloop tracking system", Proc. SPIE 6758, 67580E (2007).

[5] E. Bosman, J. Missinne, B. Van Hoe, G. Van Steenberge, S. Kalathimekkad, J. Van Erps, I. Milenkov, K. Panajotov, T. Van Gijseghem, P. Dubruel, H. Thienpont and P. Van Daele, "Ultrathin Optoelectronic Device Packaging in Flexible Carriers", IEEE Journal of Selected Topics in Quantumelectronics, 17(3), 617-628 (2011).

[6] B. Van Hoe, E. Bosman, J. Missinne, S. Kalathimekkad, G. Van Steenberge, P. Van Daele, "Novel coupling and packaging approaches for optical interconnects", Proc. SPIE (Photonics West 2012), to be published.

[7] Shizhuo Yin, Paul B. Ruffin, Francis T.S. Yu, "Fiber optic sensors", CRC Press, 111-126 (2008).

[8] PHOSFOS, Photonic Skins for Optical Sensing, http://www.phosfos.eu. 